\title{
SPECTRAL PROPERTIES OF MICROSTRUCTURE AND VERY SHORT SPIKES IN PULSAR RADIO EMISSION
}

\author{
A. Yu. Novikov and V. A. Soglasnov \\ Institute for Space Research, Acaderny of Sciences
}

\section{Historical introduction}

Our primordial item ( 10 years ago) was to use short "unresolved" spikes, which are seen within individual pulses in some pulsars after dispersion removal (Hankins 1971) for precise measurements of dispersion measure (DM). For this purpose we performed three-frequency observations in the 60-102 MHz frequency range for PSR 0950+08, $0809+74$ and $1133+16$ (Novikov et al. 1983). The result was that "classical" mirrostructure (with 0.2 , 1.2 , and $0.55-\mathrm{ms}$ time scales, respectively) is well correlated over this frequency range, but those bursts with a shorter duration $(\sim 100 \mu \mathrm{s})$ do not show any correlation. In particular, no coincidence of the short "unresolved" spikes at two different frequencies was detected. On the basis of these results we reduced the frequency spacing to $1-2 \mathrm{MHz}$, but the result was the same (Popov et al. 1985). Our next step was to divide the total $100-\mathrm{kHz}$ band, which was used in our microstructure observations, into two $50 . \mathrm{kHz}$ parts and correlate the pulse in each part. These results were again negative for short scale phenomena, including short time periodicity (Kuz'min 1989b).

As a last attempt to understand this strange, in our view, behavior - that is, the narrow frequency band and short time scales for this phenomena--we made a complex analysis of the single pulse emission of PSR $0809+74$, recorded at $102.5 \mathrm{MHz}$ in a $100-\mathrm{kHz}$ frequency on the $\mathrm{BSA}$ radio telescope at Pushchino in 1980.

The processing procedure consisted of several steps. First dispersion distortion was removed before detection over the full $100-\mathrm{kHz}$ band. After detection the signal was smoothed with an $80-\mu$ s time constant. A radio frequency spectrum for individual subpulses was also obtained. The total $100-\mathrm{kHz}$ band was divided into $1612.5 \mathrm{kHz}$ bands spaced by $6.25 \mathrm{kHz}$, so adjacent bands were semi-overlapped; only 10 such narrow bands were placed within the flat part of the receiver band $(68.5 \mathrm{kHz})$. In eaci such band the signal time resolution was restored to $80 \mu \mathrm{s}$.

An example of the subpulses seen in different $12.5-\mathrm{kHz}$ bands is shown in figure la. Crosscorrelation functions (CCF's) were computed for each of these "narrow band subpulses" for all combinations of the bands. The CCF's were then averaged by frequency difference-that is, for the $0 \mathrm{kHz}$ spacing there were 10 CCF's (ACF's), for $6.25 \mathrm{kHz}$, 9 CCF's (semioverlapped channels), for $12.5 \mathrm{kHz}, 8$ CCF's and so on. (See figure 1b.). This procedure was carried out for $\sim 100$ strong pulses (the mean CCF's are shown in figure 1c.) and for "clean sky" for comparison.

Mean CCF's show "classical" microstructure features with time scales of $1.2 \mathrm{~ms}$ and no features with smaller scales. Some of the individual subpulses $(\sim 20 \%)$ show narrower features, but these are not observed, as a rule, at other frequency spacings; thus short time microstructure, if present, varies chaotically from band to band.

The spectra of subpulses show frequency modulation with 4 to $7-\mathrm{kHz}$ features (figure 1), which corresponds to a 140 to $250-\mu$ s scale in the time domain-that is, to the "short microstructure". These spectral features changed rapidly from pulse to pulse and even for different subpulses within one pulse. Note that features with scales $\sim 0.8 \mathrm{kHz}$ which correspond to the $1.2 \mathrm{-ms}$ microstructure are not seen in figure 1 owing to the $1.28 \mathrm{kHz}$ smooth ing. The mean spectrum integrated over 100 subpulses is featureless, except for a slow rise from midband due to interstellar scintillation.

\section{Unresolved spikes}

When the pulsar signal in narrow bands was studied, we detected strong "unresolved" spikes which appear simultaneously in some bands. The term "unresolved" spike means a short pulse with duration equal to the time resolution of the band $t_{\text {unres }}=80 \mu \mathrm{s}$. Figure 3 demonstrates the phenomenon of "unresolved" spikes.

The left side of figure 3 shows the radio spectrum of the subpulses in the total receiver band. The center shows the pulse windows with $20.48-\mathrm{ms}$ duration as in figure 1 . The eleventh plot is the pulsar signal in the whole $100-\mathrm{kHz}$ band and is the sum of the ten narrow-band pulsar signals. The right side gives two narrow windows with $5.12-\mathrm{ms}$ duration and higher time resolution.

It is clearly seen from figure 3 that the spikes do 


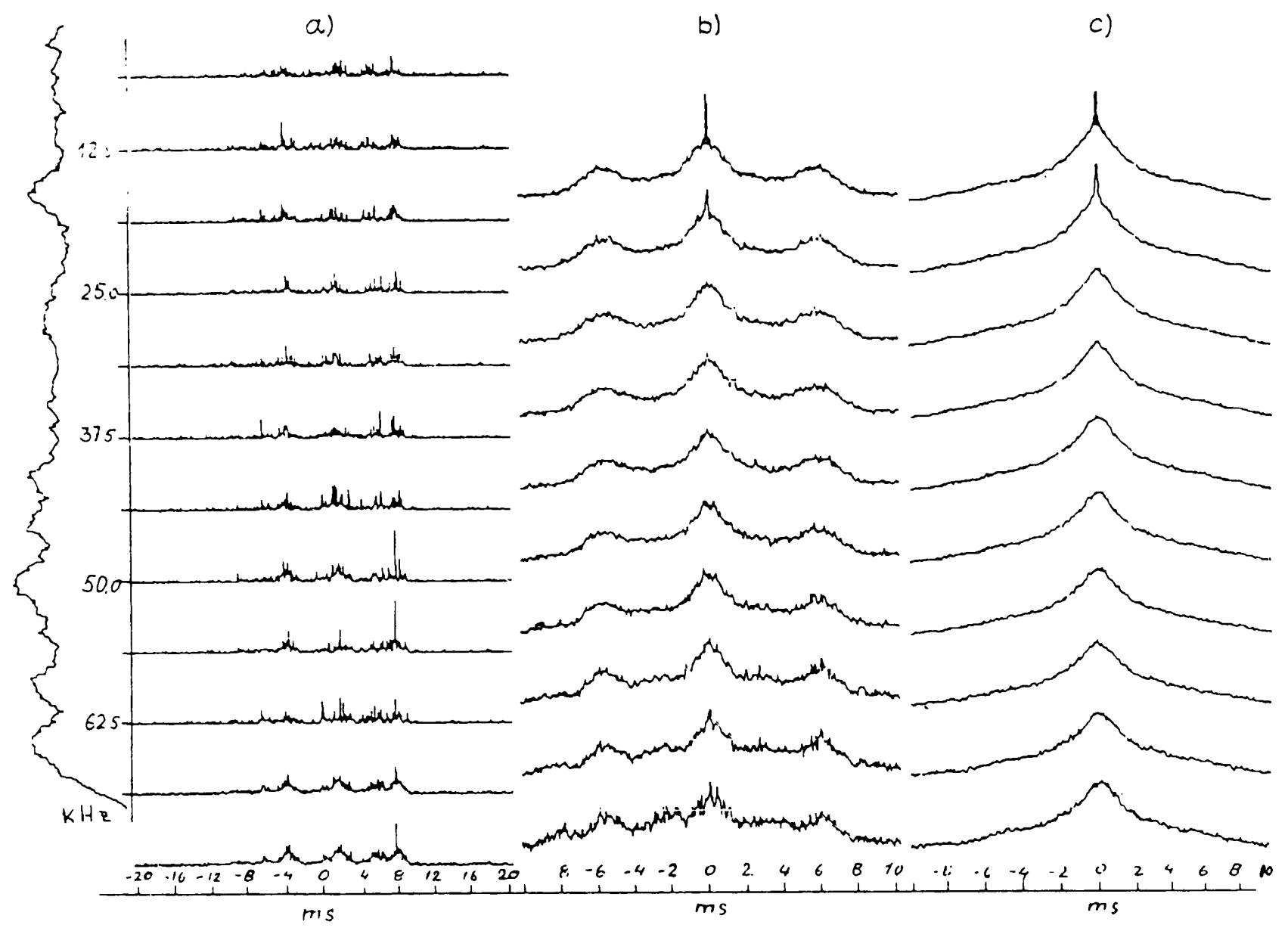

Figure 1 Subpulse intensity vs. frequency across a 100-kHz band. a) The first ten plots represent subpulses in different $12.5-\mathrm{kHz}$ tands with a time resolution of $80 \mu \mathrm{s}$; the frequency spacing between adjacent bands is $6.25 \mathrm{kHz}$. The subpulse spectrum in the total $100-\mathrm{kHz}$ band is shown in the vertical graph on the left; its spectral resolution is $1.28 \mathrm{kHz}$. The lowest plot is the sum of the $1012.5 \cdot \mathrm{kHz}$ bands. b) Cross-correlation functions (CCF's) of subpulse intensity between different $12.5-\mathrm{kHz}$ bands, averaged according to frequency spacing: First $0-\mathrm{kHz}$ spacing (ACF), 10 ACF's are averaged; second $6.25-\mathrm{kHz}$ spacing (semi-overlapping bands), 9 CCF's are averaged; third 12.5-kHz spacing, 8 CCF's are averaged; and so on. c) The same CCF's averaged over 75 individual subpulses.

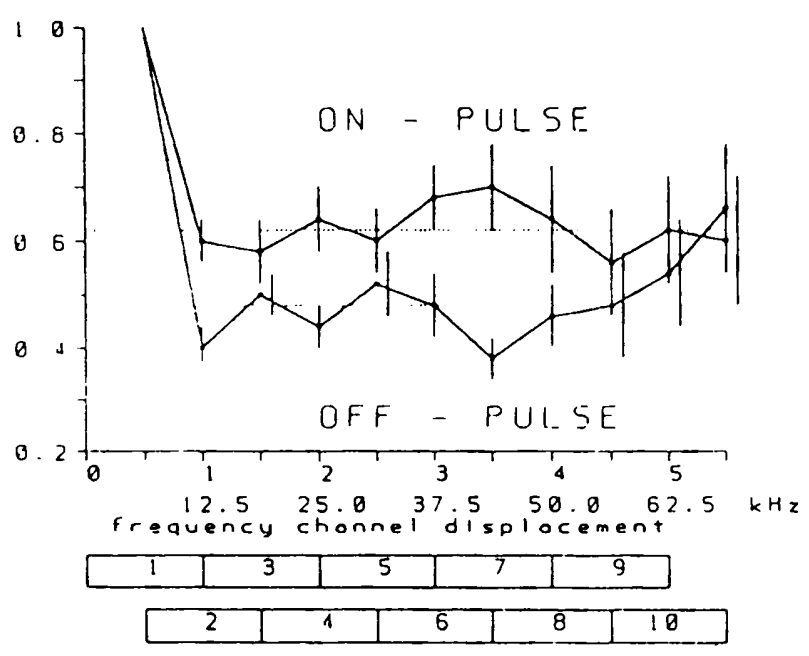

Figure 2 Distribution of pulses which appear simultaneously at different frequency channels vs. frequency displacement

not appear simultaneously in all narrow bands. To estimate the statistical character of the spikes appearing at the same phase in different narrow bands, we calculated the distribution of these events vs. the displacement of each narrow frequency channel. We analyzed about 1500 such cases, consisting both of on-pulse and off-pulse signals. Both distributions were normalized by the number of simultaneous pulses in semi-overlapping bands. For this preliminary estimation we selected 26 subpulses with unresolved spikes. The distribution shows that simultaneous "unresolved" spikes are more frequent in the on-pulse signal at a level of $28 \pm 3 \%$.

Note that there is no dependence of the simultaneous appearance of spikes as a function of frequency displacement within the error level.

\section{Conclusions}

We suppose that this short time scale microstructure may be explained in the following way. The 


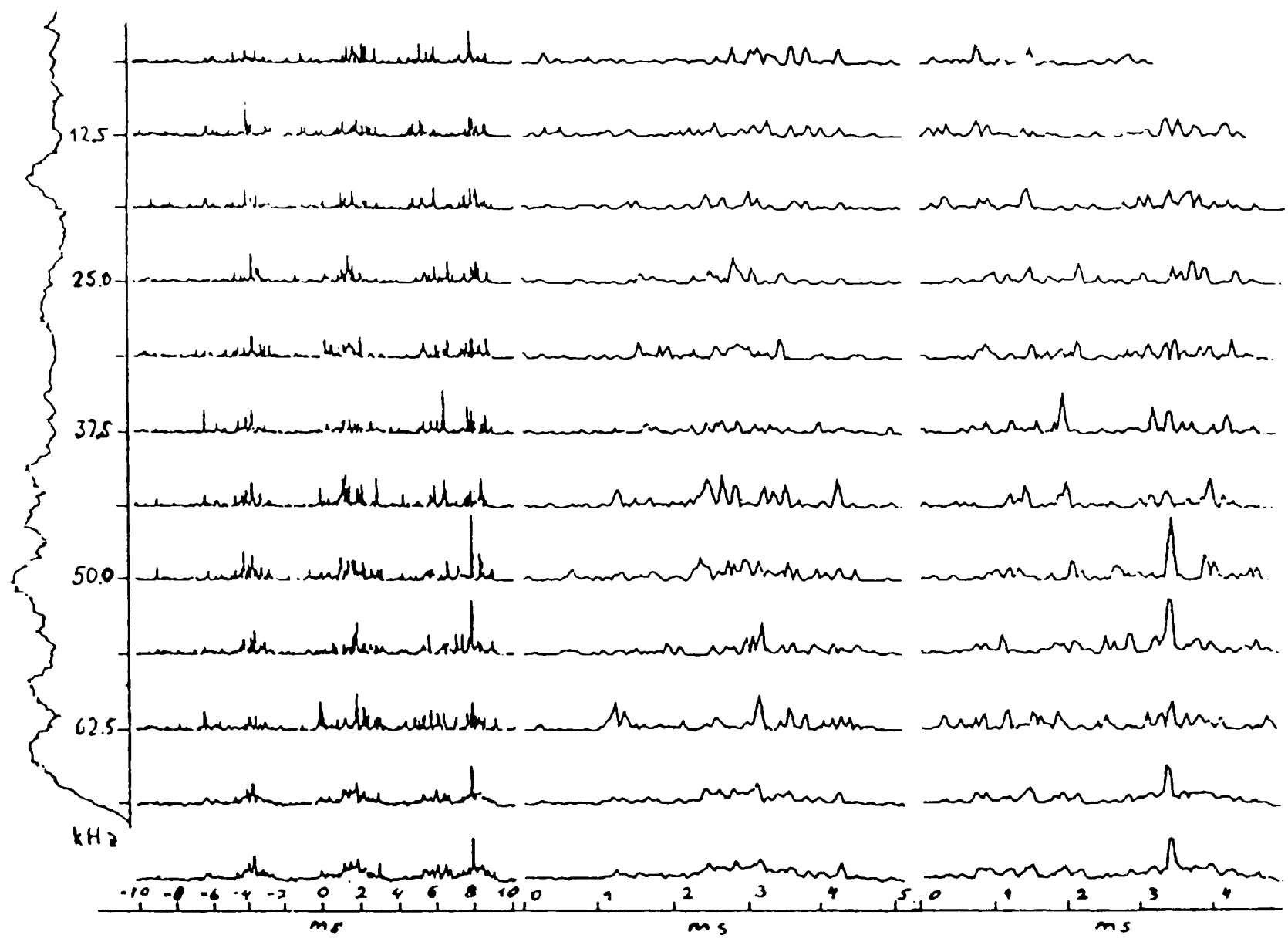

Figure 3 Examples of "unresolved" spikes in narrow frequency bands for one subpulse of PSR 0809+74. Two on-pulse windows with high time resolution are shown.

essential property of pulsar radio emission is its frequency modulation with a characteristic width of frequency features, $v_{c}$, which are randomly located in frequency. If the received frequency bandwidth $B$ is larger than $v_{c}$ time structure with a scale $\sim 1 / v_{c}$ arises; if several features are inside $B$, additional time modulation with a scale $<1 / v_{\mathrm{c}}$ will be observed, in particular as short "unresolved" spikes. This short time scale microstructure varies rapidly when the frequency band shifts more than $v_{c}$, and it also varies from pulse to pulse, because the frequency features are short-lived structures. But the frequency range in which the modulation phenomenon exists may itself be rather wide, so that some kind of wide band "quasi-correlated" short time-scale microstructure will be observed. In order to further verify this scheme, we plan to perform a similar analysis in the frequency domain. 


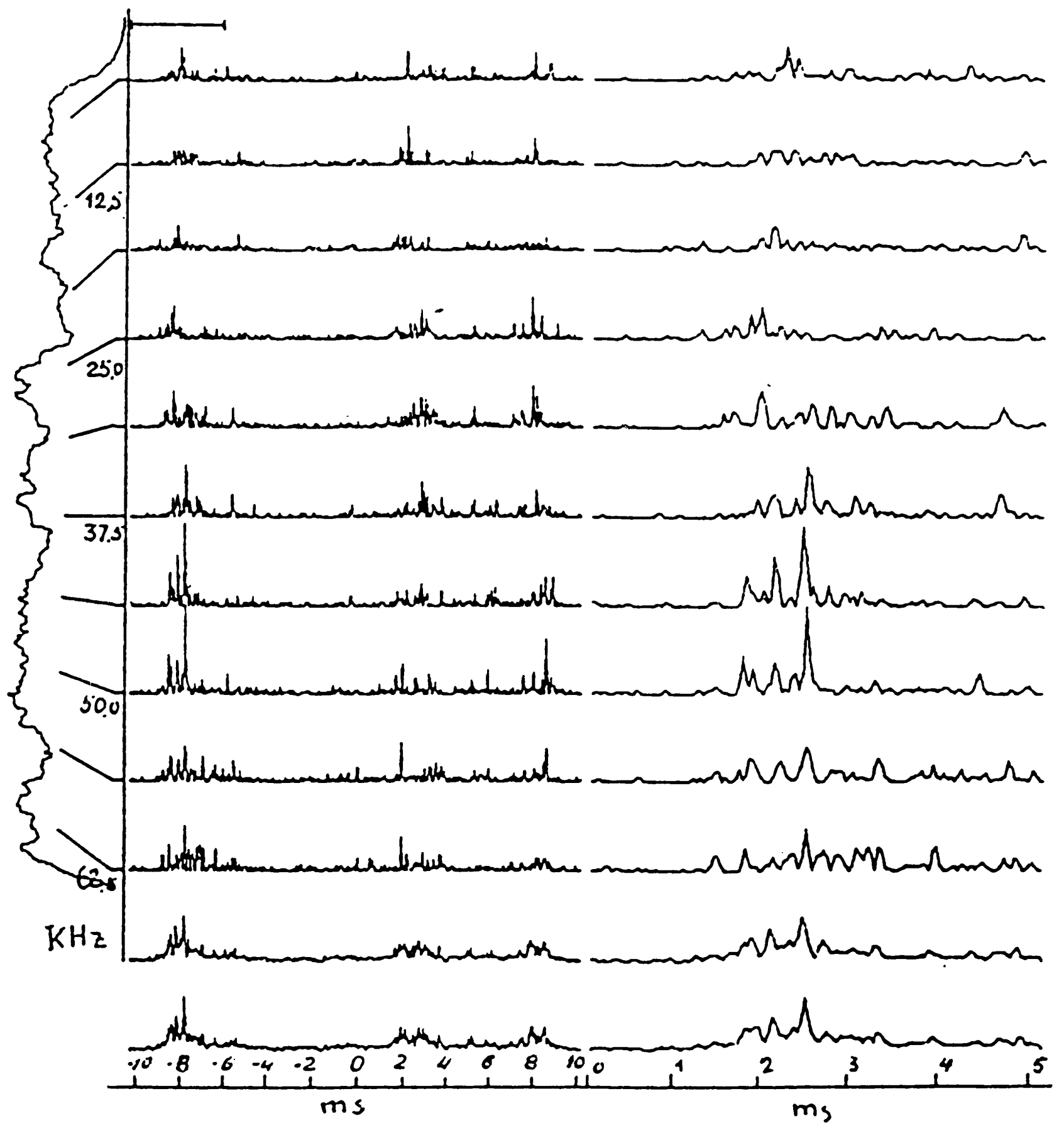

Figure 4 Example of the appearance of a triple "unresolved" spike in different narrow bands. 